\title{
Trapezoidal Intuitionistic Fuzzy Aggregation Operator Based on Choquet Integral and Its Application to Multi-Criteria Decision-Making Problems
}

\begin{abstract}
The Choquet integral can serve as a useful tool to aggregate interacting criteria in an uncertain environment. In this paper, a trapezoidal intuitionistic fuzzy aggregation operator based on the Choquet integral is proposed for multi-criteria decision-making problems. The decision information takes the form of trapezoidal intuitionistic fuzzy numbers and both the importance and the interaction information among decision-making criteria are considered. On the basis of the introduction of trapezoidal intuitionistic fuzzy numbers, its operational laws and expected value are defined. A trapezoidal intuitionistic fuzzy aggregation operator based on the Choquet integral is then defined and some of its properties are investigated. A new multi-criteria decision-making method based on a trapezoidal intuitionistic fuzzy Choquet integral operator is proposed. Finally, an illustrative example is used to show the feasibility and availability of the proposed method.
\end{abstract}

Keywords: multi-criteria decision making, trapezoidal intuitionistic fuzzy numbers, Choquet integral, fuzzy measure, aggregation operator

\section{Introduction}

In the real decision-making problems, information available to decision-makers is often vague and imprecise. It is, thus, necessary to study fuzzy multi-criteria decisionmaking problems with an intuitionistic fuzzy setting. The Intuitionistic Fuzzy Set (IFS) theory, which was first introduced by Atanassov (1986), provides us with a powerful tool to tackle uncertain and vague information

Manuscript received March 25, 2015; accepted September 11, 2015

Xi-hua Li ( $\bowtie)$, Xiao-hong Chen

Business School of Central South University; Collaborative Innovation Center of Resource-conserving \& Environment-friendly Society and Ecological Civilization, Changsha 410083, China

Email: xihuali@126.com in real applications by allocating each element in intuitionistic fuzzy sets a membership degree and a nonmembership degree. Since its appearance, it has received more and more attention. Recently, IFS theory has widely been applied to multi-criteria decision-making problems. For example, Chen and Tan (1994), Hong and Choi (2000), Li (2005), Xu and Yager (2006, 2008), Liu and Wang (2007), Xu (2007), Li, Wang, Liu, and Shan (2008), Wei (2009), Ye (2009, 2010), Wu and Zhang (2011) presented some new methods for handling multi-criteria decisionmaking problems based on intuitionistic fuzzy sets. However, the intuitionistic fuzzy set can only express the extent to which a criterion does or does not belong to a fuzzy concept "Excellence" or "Good" and IFS only use discrete domains. Fuzzy numbers are a special case of fuzzy sets and are of importance for fuzzy multi-attribute decision-making problems (Abbasbandy \& Hajjari, 2009; Asady \& Zendehnam, 2007; Dubois \& Prade, 1980; Vencheh \& Allame, 2010). Nehi and Maleki (2005) introduced the intuitionistic trapezoidal fuzzy numbers as the extension of intuitionistic triangular fuzzy numbers and proved some operation for them. The triangular intuitionistic fuzzy numbers and trapezoidal intuitionistic fuzzy numbers are the extension of intuitionistic fuzzy sets in another way, which extends discrete set to continuous set, and they are the extending of fuzzy numbers (Wang \& Zhang, 2009; Ye, 2011).

Furthermore, all above mentioned studies only consider the situations that all of the elements in intuitionistic fuzzy sets are independent. However, for real decision-making problems, there always exist interactive characteristics among the elements in an uncertain situation. The Choquet integral is recognized as a useful tool to aggregate interacting criteria under uncertainty (Choquet, 1954). The aggregation based on the Choquet integral can deal with the decision information that may be correlative with each other. So, it is necessary to use the Choquet integral to overcome the limitation of independence assumption among criteria in multi-criteria decision making. Xu (2010) and Tan and Chen (2010) have paid attention to 
the advantage of using the Choquet integral in real applications, and proposed some intuitionistic fuzzy Choquet integral operators using intuitionistic fuzzy sets.

Motivated by the advantages of intuitionistic trapezoidal fuzzy numbers and the Choquet integral in multi-criteria decision making, in this paper we propose a trapezoidal intuitionistic fuzzy aggregation operator based on the Choquet integral for multi-criteria decision-making problems. The decision information takes the form of trapezoidal intuitionistic fuzzy numbers and both the importance and interaction information among decision making criteria are considered.

This paper is organized as follows. In section 2, we define the trapezoidal intuitionistic fuzzy numbers and introduce some operational laws on trapezoidal intuitionistic fuzzy numbers. In section 3 , based on a review of the Choquet integral, a trapezoidal intuitionistic fuzzy aggregation operator based on the Choquet integral is defined and some of its properties are investigated. In section 4, a kind of multi-criteria decision making method based on trapezoidal intuitionistic fuzzy Choquet integral operator is proposed. In section 5, an illustrative example shows the feasibility and availability of the proposed method. Finally, some concluding remarks are drawn in Section 6.

\section{Trapezoidal intuitionistic fuzzy numbers}

In this section, we briefly review some definitions related to intuitionistic fuzzy sets, trapezoidal intuitionistic fuzzy numbers, then the expected values and some operational laws of trapezoidal intuitionistic fuzzy numbers are defined.

Definition 1. Let $X$ be a universe of discourse. Then an intuitionistic fuzzy set $A$ in $X$ is given by [1]

$$
A=\left\{\left\langle X, \mu_{A}(x), v_{A}(x) \mid x \in X\right\rangle\right\}
$$

where $\mu_{A}(x): X \rightarrow[0,1], v_{A}(x): X \rightarrow[0,1]$ with the condition

$$
0 \leqslant \mu_{A}(x)+v_{A}(x) \leqslant 1 \text {, for all } x \in X \text {. }
$$

The numbers $\mu_{A}(x)$ and $v_{A}(x)$ denote the degree of membership and non-membership of the element $x$ to the set $A$, respectively. In addition, the degree of hesitancy of $x$ can be computed as follows:

$$
\pi_{A}(x)=1-\mu_{A}(x)-v_{A}(x) \text { for all } x \in X
$$

Obviously, if $\pi_{A}(x)=0$, then intuitionistic fuzzy set reduces to a fuzzy set.

A trapezoidal intuitionistic fuzzy number is a special intuitionistic fuzzy set. According to the studies of Nehi and Maleki (2005) and Wang and Zhang (2009), we introduce the definition of a trapezoidal intuitionistic fuzzy number as follows.

Definition 2. A trapezoidal intuitionistic fuzzy number $\tilde{a}$ with parameters $b_{1} \leqslant a_{1} \leqslant b_{2} \leqslant a_{2} \leqslant a_{3} \leqslant b_{3} \leqslant a_{4} \leqslant b_{4}$ is denoted as $\tilde{a}=\left\langle\left(a_{1}, a_{2}, a_{3}, a_{4}\right),\left(b_{1}, b_{2}, b_{3}, b_{4}\right) ; \mu_{\tilde{a}}, v_{\tilde{a}}\right\rangle$ on a real number set $R$, and its membership is defined as follows:

$$
\mu_{\tilde{a}}(x)=\left\{\begin{array}{l}
f_{\tilde{a}}^{L}(x), a_{1} \leqslant x<a_{2} \\
\mu_{\tilde{a}}, a_{2} \leqslant x \leqslant a_{3} \\
f_{\tilde{a}}^{R}(x), a_{3}<x \leqslant a_{4} \\
0, \text { otherwise }
\end{array}\right.
$$

whereas its non-membership function is defined as follows:

$$
v_{\tilde{a}}(x)=\left\{\begin{array}{l}
h_{\tilde{a}}^{L}(x), b_{1} \leqslant x<b_{2} \\
v_{\tilde{a}}, b_{2} \leqslant x \leqslant b_{3} \\
h_{\tilde{a}}^{R}(x), b_{3}<x \leqslant b_{4} \\
1, \text { otherwise }
\end{array}\right.
$$

where $f_{\tilde{a}}^{L}(x), f_{\tilde{a}}^{R}(x), h_{\tilde{a}}^{L}(x), h_{\tilde{a}}^{R}(x)$ are the left and the right basis functions of the membership function and nonmembership function, respectively, and

$$
\begin{gathered}
f_{\tilde{a}}^{L}(x)=\frac{x-a_{1}}{a_{2}-a_{1}} \mu_{\tilde{a}}, f_{\tilde{a}}^{R}(x)=\frac{a_{4}-x}{a_{4}-a_{3}} \mu_{\tilde{a}} \\
h_{\tilde{a}}^{L}(x)=\frac{b_{2}-x+v_{\tilde{a}}\left(x-b_{1}\right)}{b_{2}-b_{1}} \\
h_{\tilde{a}}^{R}(x)=\frac{x-b_{3}+v_{\tilde{a}}\left(b_{4}-x\right)}{b_{4}-b_{3}}
\end{gathered}
$$

$0 \leqslant \mu_{\tilde{a}} \leqslant 1,0 \leqslant v_{\tilde{a}} \leqslant 1, \mu_{\tilde{a}}+v_{\tilde{a}} \leqslant 1$.

We call $\pi_{\tilde{a}}=1-\mu_{\tilde{a}}-v_{\tilde{a}}$ the degree of hesitancy of a trapezoidal intuitionistic fuzzy number $\tilde{a}$. Obviously, if $b_{2}=$ $b_{3}$ (hence $a_{2}=a_{3}$ ) in a trapezoidal intuitionistic fuzzy number $\tilde{a}$, then $\tilde{a}$ reduces to a triangular intuitionistic fuzzy number.

A useful tool to deal with fuzzy numbers is the $\alpha$-cuts. Similarly, Nehi and Maleki (2005) and Ye (2011) proposed $\alpha$-cuts in the case of a intuitionistic fuzzy number. Motivated by these studies, we propose $\alpha$-cuts in the case of trapezoidal intuitionistic fuzzy numbers.

Definition 3. Let $\tilde{a}$ be a trapezoidal intuitionistic fuzzy number. The corresponding $\alpha$-cuts, i.e., $\left(\tilde{a}^{+}\right)_{\alpha}$ and $(\tilde{a})_{\alpha}$, are, respectively, defined as

$$
\begin{gathered}
\left(\tilde{a}^{+}\right)_{\alpha}=\left\{x \in R \mid \mu_{\tilde{a}}(x) \geqslant \alpha\right\} \\
\left(\tilde{a}^{-}\right)_{\alpha}=\left\{x \in R \mid 1-v_{\tilde{\alpha}}(x) \geqslant \alpha\right\}
\end{gathered}
$$

In view of the definition, each $\alpha$-cut is a closed interval. Hence, we have $\left(\tilde{a}^{+}\right)_{\alpha}=\left[\tilde{a}_{L}^{+}(\alpha), \tilde{a}_{U}^{+}(\alpha)\right]$ and $\left(\tilde{a}^{-}\right)_{\alpha}=\left[\tilde{a}_{L}^{-}(\alpha)\right.$, $\left.\tilde{a}_{U}(\alpha)\right]$, where

$$
\tilde{a}_{L}^{+}(\alpha)=\inf \left(x \in R \mid \mu_{\tilde{a}}(x) \geqslant \alpha\right)
$$




$$
\begin{gathered}
\tilde{a}_{U}^{+}(\alpha)=\sup \left(x \in R \mid \mu_{\tilde{a}}(x) \geqslant \alpha\right) \\
\tilde{a}_{L}^{-}(\alpha)=\inf \left(x \in R \mid 1-v_{\tilde{a}}(x) \geqslant \alpha\right) \\
\tilde{a}_{U}^{-}(\alpha)=\sup \left(x \in R \mid 1-v_{\tilde{a}}(x) \geqslant \alpha\right)
\end{gathered}
$$

Another important concept is the expected value of a trapezoidal intuitionistic fuzzy number, which is defined as follows:

Definition 4. Let $\tilde{a}$ be a trapezoidal intuitionistic fuzzy number in the set of real numbers $R$. Then, the expected value $E V(\tilde{a})$ of $\tilde{a}$ is the center of the expected interval of $\tilde{a}$, which is defined by

$$
E V(\tilde{a})=\left[E V_{*}(\tilde{a})+E V^{*}(\tilde{a})\right] / 2
$$

where $\left[E V_{*}(\tilde{a}), E V^{*}(\tilde{a})\right]$ is the expected interval, $E V_{*}(\tilde{a})=\frac{\left(\int_{0}^{\mu_{\tilde{a}}} \tilde{a}_{L}^{+}(\alpha) \mathrm{d} \alpha+\int_{0}^{1-v_{\tilde{a}}} \tilde{a}_{L}^{-}(\alpha) \mathrm{d} \alpha\right)}{2}$, and $E V_{*}(\tilde{a})$ $=\frac{\left(\int_{0}^{\mu_{\tilde{a}}} \tilde{a}_{U}^{+}(\alpha) \mathrm{d} \alpha+\int_{0}^{1-v_{\tilde{a}}} \tilde{a}_{U}^{-}(\alpha) \mathrm{d} \alpha\right)}{2}$.

Theorem 1. Let $\tilde{a}=\left\langle\left(a_{1}, a_{2}, a_{3}, a_{4}\right),\left(b_{1}, b_{2}, b_{3}, b_{4}\right) ; \mu_{\tilde{a}}, v_{\tilde{a}}\right\rangle$ be a trapezoidal intuitionistic fuzzy number in the set of real numbers $R$. Thus, its expected value is obtained by

$$
\begin{aligned}
& E V(\tilde{a})= \\
& {\left[\mu_{\tilde{a}}\left(a_{1}+a_{2}+a_{3}+a_{4}\right)+\left(1-v_{\tilde{a}}\right)\left(b_{1}+b_{2}+b_{3}+b_{4}\right)\right] / 8}
\end{aligned}
$$

Especially, if $\mu_{\tilde{a}}=1$ (hence $v_{\tilde{a}}=0$ and $\left(a_{1}, a_{2}, a_{3}, a_{4}\right)=\left(b_{1}\right.$, $\left.b_{2}, b_{3}, b_{4}\right)$, then

$$
E V(\tilde{a})=\left(a_{1}+a_{2}+a_{3}+a_{4}\right) / 4
$$

Thus, the above equation reduces to the expected value of a trapezoidal fuzzy number.

To compare two trapezoidal intuitionistic fuzzy numbers, Wang and Zhang (2009) proposed score function and accuracy function of two trapezoidal intuitionistic fuzzy numbers.

Definition 5. Let $\tilde{a}_{1}$ and $\tilde{a}_{2}$ be two trapezoidal intuitionistic fuzzy numbers, $S\left(\tilde{a}_{1}\right)=E V\left(\tilde{a}_{1}\right) \times\left(\mu_{\tilde{a}_{1}}-v_{\tilde{a}_{1}}\right)$ and $S\left(\tilde{a}_{2}\right)=E V\left(\tilde{a}_{2}\right) \times\left(\mu_{\tilde{a}_{2}}-v_{\tilde{a}_{2}}\right)$ be the score functions of $\tilde{a}_{1}$ and $\tilde{a}_{2}$, respectively. Let $H\left(\tilde{a}_{1}\right)=E V\left(\tilde{a}_{1}\right) \times\left(\mu_{\tilde{a}_{1}}+v_{\tilde{a}_{1}}\right)$ and $H$ $\left(\tilde{a}_{2}\right)=E V\left(\tilde{a}_{2}\right) \times\left(\mu_{\tilde{a}_{2}}+v_{\tilde{a}_{2}}\right)$ be the accuracy functions of $\tilde{a}_{1}$ and $\tilde{a}_{2}$, respectively, then

If $S\left(\tilde{a}_{1}\right)>S\left(\tilde{a}_{2}\right)$, then $\tilde{a}_{1}>\tilde{a}_{2}$;

If $S\left(\tilde{a}_{1}\right)=S\left(\tilde{a}_{2}\right)$, then

(1) If $H\left(\tilde{a}_{1}\right)>H\left(\tilde{a}_{2}\right)$, then $\tilde{a}_{1>} \tilde{a}_{2}$;

(2) If $H\left(\tilde{a}_{1}\right)=H\left(\tilde{a}_{2}\right)$ then $\tilde{a}_{1}=\tilde{a}_{2}$.

Definition 6. Let $\tilde{a}_{1}$ and $\tilde{a}_{2}$ be two trapezoidal intuitionistic fuzzy numbers

$$
\begin{aligned}
& \tilde{a}_{1}=\left\langle\left(a_{11}, a_{12}, a_{13}, a_{14}\right),\left(b_{11}, b_{12}, b_{13}, b_{14}\right) ; \mu_{\tilde{a}_{1}}, v_{\tilde{a}_{1}}\right\rangle \\
& \tilde{a}_{2}=\left\langle\left(a_{21}, a_{22}, a_{23}, a_{24}\right),\left(b_{21}, b_{22}, b_{23}, b_{24}\right) ; \mu_{\tilde{a}_{2}}, v_{\tilde{a}_{2}}\right\rangle
\end{aligned}
$$

then we have

$$
\begin{aligned}
\tilde{a}_{1} \oplus \tilde{a}_{2}= & \left\langle\left( a_{11}+a_{21}, a_{12}+a_{22}, a_{13}+a_{23}, a_{14}\right.\right. \\
& \left.\left.+a_{24}\right)\right\rangle,\left(b_{11}+b_{21}, b_{12}+b_{22}, b_{13}\right. \\
& \left.+b_{23}, b_{14}+b_{24}\right) ; \mu_{\tilde{a}_{1}} \\
& +\mu_{\tilde{a}_{2}}-\mu_{\tilde{a}_{1}} \mu_{\tilde{a}_{2}}, v_{\tilde{a}_{1}} v_{\tilde{a}_{2}},
\end{aligned}
$$

$$
\begin{aligned}
\lambda \tilde{a}_{1}= & \left\langle\left(\lambda a_{11}, \lambda a_{12}, \lambda a_{13}, \lambda a_{14}\right),\left(\lambda b_{11}, \lambda b_{12}, \lambda b_{13}, \lambda b_{14}\right) ;\right. \\
& \left.1-\left(1-\mu_{\tilde{a}_{1}}\right)^{\lambda}, v_{\tilde{a}_{1}}^{\lambda}\right\rangle, \lambda>0 .
\end{aligned}
$$

Proposition 1. Let $\tilde{a}_{1}$ and $\tilde{a}_{2}$ be two trapezoidal intuitionistic fuzzy numbers, and $\tilde{a}_{3}=\tilde{a}_{1} \oplus \tilde{a}_{2}, \quad \tilde{a}_{4}=\lambda \tilde{a}_{1}$, where $\lambda$ is a positive real number; then both $\tilde{a}_{3}$ and $\tilde{a}_{4}$ are also trapezoidal intuitionistic fuzzy numbers.

Proposition 2. Let $\tilde{a}_{1}$ and $\tilde{a}_{2}$ be two trapezoidal intuitionistic fuzzy numbers and $\lambda_{1}$ and $\lambda_{2}$ are positive real numbers, one then obtains

$$
\begin{gathered}
\tilde{a}_{1} \oplus \tilde{a}_{2}=\tilde{a}_{2} \oplus \tilde{a}_{1} \\
\lambda_{1} \tilde{a}_{1} \oplus \lambda_{2} \tilde{a}_{1}=\left(\lambda_{1}+\lambda_{2}\right) \tilde{a}_{1} \\
\lambda_{1} \tilde{a}_{1} \oplus \lambda_{1} \tilde{a}_{2}=\lambda_{1}\left(\tilde{a}_{1}+\tilde{a}_{2}\right)
\end{gathered}
$$

According to the operational laws of Definition 3, Wang and Zhang (2009) extended the weighted averaging operator to trapezoidal intuitionistic fuzzy numbers, and proposed the trapezoidal intuitionistic fuzzy weighted averaging operator, which is defined as follows:

Definition 7. Let $\tilde{a}_{i}(i=1,2, \ldots, n)$ be a collection of trapezoidal intuitionistic fuzzy numbers, and $w=\left(w_{1}\right.$, $\left.w_{2}, \ldots, w_{n}\right)^{\mathrm{T}}$ be the weight vector of $\tilde{a}_{i}$, we then define the trapezoidal intuitionistic fuzzy weighted averaging operator (TIFWA) as

$$
\operatorname{TIFWA}\left(\tilde{a}_{1}, \tilde{a}_{2}, \ldots, \tilde{a}_{n}\right)=w_{1} \tilde{a}_{1} \oplus w_{2} \tilde{a}_{2} \oplus \ldots \oplus w_{n} \tilde{a}_{n}
$$

Motivated by the order weighted averaging operator (OWA) proposed by Yager (1988), we extended the OWA operator to trapezoidal intuitionistic fuzzy numbers and propose a trapezoidal intuitionistic fuzzy order weighted averaging operator(TIFOWA), which is defined as follows:

Definition 8 . Let $\tilde{a}_{i}(i=1,2, \ldots, n)$ be a collection of trapezoidal intuitionistic fuzzy numbers, and $w=\left(w_{1}\right.$, $\left.w_{2}, \ldots, w_{n}\right)^{\mathrm{T}}$ be the weight vector associated with the TIFOWA, and then TIFOWA can be computed by the following expression:

$$
\operatorname{TIFWA}\left(\tilde{a}_{1}, \tilde{a}_{2}, \ldots, \tilde{a}_{n}\right)=w_{1} \tilde{a}_{(1)} \oplus w_{2} \tilde{a}_{(2)} \oplus \ldots \oplus w_{n} \tilde{a}_{(n)}
$$

where $(i)$ indicates a permutation on $X$ such that $\tilde{a}_{(1)} \leqslant \tilde{a}_{(2)} \leqslant \ldots \leqslant \tilde{a}_{(n)}$. 


\section{Trapezoidal intuitionistic fuzzy Choquet integral operator}

\subsection{Fuzzy measure and the Choquet integral}

The philosophy of the Choquet integral was first introduced in capacity theory and used as a (fuzzy) integral with respect to a fuzzy measure proposed by Höhle (1982) and then rediscovered later by Murofushi and Sugeno (1989, 1991). The Choquet integral is defined to integrate functions with respect to the fuzzy measures (Murofushi \& Sugeno, 1989).

As for the multi-criteria decision-making problems, in the Choquet integral model with criteria dependent, a fuzzy measure for criteria is used to define a weight on each combination of classifiers, thus making it possible to model the interaction among criteria. Fuzzy measure was first introduced by Sugeno (1974), which makes a monotonicity instead of additive property. The definitions of fuzzy measures and the Choquet integral are as follows (Murofushi \& Sugeno, 1989).

Definition 9. Let $X$ be a finite set, and $P(X)$ be the power set of $X$. A fuzzy measure on $X$ is a set function $\psi: P(X) \rightarrow$ $[0,1]$ if the following conditions hold:

(1) $\psi(\varphi)=0, \psi(X)=1$ (boundary conditions);

(2) If $A, B \in P(X)$ and $A \subseteq B$ then $\mu(A) \leqslant \mu(B)$ (monotonicity).

$\psi(C)$ in Definition 9 can be viewed as a measure on the grade of subjective importance of decision criteria set $C$. In addition to the usual weights on criteria taken separately, weights on any combination of criteria are also defined, as follows.

Definition 10 . Let $\psi$ be a fuzzy measure on $X$. The Choquet integral of function $f: X \rightarrow R$ with respect to $\mu$ is defined as

$$
\begin{gathered}
C_{\psi}(f)=\sum_{i=1}^{n} f_{(i)}\left[\psi\left(A_{(i)}\right)-\psi\left(A_{(i+1)}\right)\right] \quad \text { (19) } \begin{array}{l}
\text { trapezoidal intuitionistic fuzzy numbers, } \\
\text { transformed into the following form by in }
\end{array} \\
\operatorname{TIFC}\left(\tilde{a}_{1}, \ldots, \tilde{a}_{n}\right)=\left\langle\begin{array}{l}
\left(\sum_{i=1}^{n} \tau_{i} a_{(i) 1}, \sum_{i=1}^{n} \tau_{i} a_{(i) 2}, \sum_{i=1}^{n} \tau_{i} a_{(i) 3}, \sum_{i=1}^{n} \tau_{i} a_{(i) 4}\right),\left(\sum_{i=1}^{n} \tau_{i} b_{(i) 1}, \sum_{i=1}^{n} \tau_{i} b_{(i) 2},\right. \\
\left.\sum_{i=1}^{n} \tau_{i} b_{(i) 3}, \sum_{i=1}^{n} \tau_{i} b_{(i) 4}\right) ; 1-\prod_{i=1}^{n}\left(1-\mu_{\tilde{a}_{(i)}}\right)^{\tau_{i}}, \prod_{i=1}^{n}\left(v_{\tilde{a}_{(i)}}\right)^{\tau_{i}}
\end{array}\right.
\end{gathered}
$$

where $(i)$ indicates a permutation on $X$ such that $f_{(1)} \leqslant f_{(2)} \leqslant \ldots \leqslant f_{(n)}$ holds. In addition, $A_{(i)}=\left\{x_{(i)}, \ldots, x_{(n)}\right\}$, $A_{(n+1)}=\phi$.

Since the Choquet integral model does not need to assume independency of one criterion from another, it can be used in nonlinear situations. The fuzzy integral of $f$ with respect to $\psi$ gives the overall evaluation of an alternative. When the criteria are independent, the fuzzy measure is additive, and the Choquet integral coincides with the weighted arithmetic average method. That is,

$$
C_{\psi}(f)=\sum_{i=1}^{n} f_{i} \psi\left(\left\{x_{i}\right\}\right)
$$

3.2 Trapezoidal intuitionistic fuzzy Choquet integral operator

Based on Definition 10, we use the Choquet integral to develop a novel operator on trapezoidal intuitionistic fuzzy numbers as follows:

Definition 11. Let $\psi$ be a fuzzy measure on $X$, and let $\tilde{a}_{i}(i=1,2, \ldots, n)$ be a collection of trapezoidal intuitionistic fuzzy numbers on $X$. The Choquet integral of $\tilde{a}_{i}$ with respect to $\psi$ is then defined as

$$
\operatorname{TIFC}\left(\tilde{a}_{1}, \ldots, \tilde{a}_{n}\right)=\bigoplus_{i=1}^{n} \tilde{a}_{(i)}\left[\psi\left(A_{(i)}\right)-\psi\left(A_{(i+1)}\right)\right]
$$

where (i) indicates a permutation on $X$ such that $\tilde{a}_{(1)} \leqslant \tilde{a}_{(2)} \leqslant \ldots \leqslant \tilde{a}_{(n)}$. In addition, $A_{(i)}=\left\{x_{(i)}, \ldots, x_{(n)}\right\}$, $A_{(n+1)}=\phi$ is satisfied.

Theorem 2. Let $\tilde{a}_{i}=\left\langle\left(a_{i 1}, a_{i 2}, a_{i 3}, a_{i 4}\right),\left(b_{i 1}, b_{i 2}, b_{i 3}, b_{i 4}\right) ; \mu_{\tilde{a}_{i}}\right.$, $\left.v_{\tilde{a}_{i}}\right\rangle(i=1,2, \ldots, n)$ be a collection of trapezoidal intuitionistic fuzzy numbers on $X$ and $\psi$ be a fuzzy measure on $X$. Thus, the value by using TIFC operator in Eq. (21) is also a trapezoidal intuitionistic fuzzy numbers, and it can be transformed into the following form by induction on $n$ : where $(i)$ indicates a permutation on $X$ such that $\tilde{a}_{(1)} \leqslant \tilde{a}_{(2)} \leqslant \ldots \leqslant \tilde{a}_{(n)}$ and $\tau_{i}=\psi\left(A_{(i)}\right)-\psi\left(A_{(i+1)}\right)$.

Proof. The first result can be easily proved by Definition
11 and Proposition 1. Now we prove Eq. (22) by using mathematical induction on $n$.

For $n=2$, according to the Definition 6, we have

$$
\begin{aligned}
& \tau_{1} \tilde{a}_{(1)}=\left\langle\left(\tau_{1} a_{(1) 1}, \tau_{1} a_{(1) 2}, \tau_{1} a_{(1) 3}, \tau_{1} a_{(1) 4}\right),\left(\tau_{1} b_{(1) 1}, \tau_{1} b_{(1) 2}, \tau_{1} b_{(1) 3}, \tau_{1} b_{(1) 4}\right) ; 1-\left(1-\mu_{\tilde{a}_{(1)}}\right)^{\tau_{1}}, v_{\tilde{a}_{(1)}}^{\tau_{1}}\right\rangle \\
& \tau_{2} \tilde{a}_{(2)}=\left\langle\left(\tau_{2} a_{(2) 1}, \tau_{2} a_{(2) 2}, \tau_{2} a_{(2) 3}, \tau_{2} a_{(2) 4}\right),\left(\tau_{1} b_{(1) 1}, \tau_{1} b_{(1) 2}, \tau_{1} b_{(1) 3}, \tau_{1} b_{(1) 4}\right) ; 1-\left(1-\mu_{\tilde{a}_{(2)}}\right)^{\tau_{2}}, v_{\tilde{a}_{(2)}}^{\tau_{2}}\right\rangle
\end{aligned}
$$


Because the following relationship holds

$$
\begin{aligned}
\tilde{a}_{1} \oplus \tilde{a}_{2}= & \left\langle\left(a_{11}+a_{21}, a_{12}+a_{22}, a_{13}+a_{23}, a_{14}+a_{24}\right),\left(b_{11}+b_{21}, b_{12}+b_{22}, b_{13}+b_{23}, b_{14}+b_{24}\right) ; \mu_{\tilde{a}_{1}}\right. \\
& \left.+\mu_{\tilde{a}_{2}}-\mu_{\tilde{a}_{1}} \mu_{\tilde{a}_{2}}, v_{\tilde{a}_{1}} v_{\tilde{a}_{2}}\right\rangle
\end{aligned}
$$

we have

$$
\operatorname{TIFC}\left(\tilde{a}_{1}, \tilde{a}_{2}\right)=\tilde{a}_{1} \tau_{1} \oplus \tilde{a}_{2} \tau_{2}=\left\langle\begin{array}{l}
\left(\sum_{i=1}^{2} \tau_{i} a_{(i) 1}, \sum_{i=1}^{2} \tau_{i} a_{(i) 2}, \sum_{i=1}^{2} \tau_{i} a_{(i) 3}, \sum_{i=1}^{2} \tau_{i} a_{(i) 4}\right),\left(\sum_{i=1}^{2} \tau_{i} b_{(i) 1}, \sum_{i=1}^{2} \tau_{i} b_{(i) 2},\right. \\
\left.\sum_{i=1}^{2} \tau_{i} b_{(i)}, \sum_{i=1}^{2} \tau_{i} b_{(i) 4}\right) ; 1-\prod_{i=1}^{2}\left(1-\mu_{\tilde{a}_{(i)}}\right)^{\tau_{i}}, \prod_{i=1}^{n}\left(v_{\tilde{a}_{(i)}}\right)^{\tau_{i}}
\end{array}\right\rangle
$$

That is, for $n=2$, Eq. (22) holds.

Assuming that for $n=k$, Eq. (20) holds, i.e.,

$$
\operatorname{TIFC}\left(\tilde{a}_{1}, \ldots, \tilde{a}_{k}\right)=\left\langle\begin{array}{l}
\left(\sum_{i=1}^{k} \tau_{i} a_{(i) 1}, \sum_{i=1}^{k} \tau_{i} a_{(i) 2}, \sum_{i=1}^{k} \tau_{i} a_{(i) 3}, \sum_{i=1}^{k} \tau_{i} a_{(i) 4}\right),\left(\sum_{i=1}^{k} \tau_{i} b_{(i) 1}, \sum_{i=1}^{k} \tau_{i} b_{(i) 2},\right. \\
\left.\sum_{i=1}^{k} \tau_{i} b_{(i) 3}, \sum_{i=1}^{k} \tau_{i} b_{(i) 4}\right) ; 1-\prod_{i=1}^{k}\left(1-\mu_{\tilde{a}_{(i)}} \tau_{i}, \prod_{i=1}^{k}\left(v_{\tilde{a}_{(i)}} \tau^{\tau_{i}}\right.\right.
\end{array}\right\rangle
$$

Then, for $n=k+1$, by Definition 6 , we have

$$
\begin{gathered}
\tau_{k+1} \tilde{a}_{(k+1)}=\left\langle\left(\tau_{k+1} a_{(k+1) 1}, \tau_{k+1} a_{(k+1) 2}, \tau_{k+1} a_{(k+1) 3}, \tau_{k+1} a_{(k+1) 4}\right),\left(\tau_{k+1} b_{(k+1) 1}, \tau_{k+1} b_{(k+1) 2}, \tau_{k+1} b_{(k+1) 3}, \tau_{k+1} b_{(k+1) 4}\right) ;\right. \\
\left.1-\left(1-\mu_{\tilde{a}_{(k+1)}}\right)^{\tau_{k+1}}, \tilde{a}_{\tilde{a}_{(k+1)}}^{\tau_{k+1}}\right\rangle
\end{gathered}
$$

According to Definition 11 and Definition 6, we have

$$
\begin{aligned}
& \operatorname{TIFC}\left(\tilde{a}_{1}, \ldots, \tilde{a}_{k+1}\right)=\bigoplus_{i=1}^{k} \tilde{a}_{i} \tau_{1} \oplus \tilde{a}_{k+1} \tau_{k+1} \\
& =\left\langle\begin{array}{l}
\left(\sum_{i=1}^{k+1} \tau_{i} a_{(i) 1}, \sum_{i=1}^{k+1} \tau_{i} a_{(i) 2}, \sum_{i=1}^{k+1} \tau_{i} a_{(i) 3}, \sum_{i=1}^{k+1} \tau_{i} a_{(i) 4}\right),\left(\sum_{i=1}^{k+1} \tau_{i} b_{(i) 1}, \sum_{i=1}^{k+1} \tau_{i} b_{(i) 2},\right. \\
\left.\sum_{i=1}^{k+1} \tau_{i} b_{(i) 3}, \sum_{i=1}^{k+1} \tau_{i} b_{(i) 4}\right) ; 1-\prod_{i=1}^{k+1}\left(1-\mu_{\tilde{a}_{(i)}}\right)^{\tau_{i}}, \prod_{i=1}^{k+1}\left(v_{\tilde{a}_{(i)}}\right)^{\tau_{i}}
\end{array}\right\rangle
\end{aligned}
$$

This means that for $n=k+1$, Eq. (22) holds. Therefore, for all $n$, Eq. (22) always holds.

In the following, we consider some special cases of TIFC operator.

Proposition 3. Let all elements $\tilde{a}_{i}(i=1,2, \ldots, n)$ in the TIFC operator be independent, thus

$$
\psi\left(a_{(i)}\right)=\psi\left(A_{(i)}\right)-\psi\left(A_{(i+1)}\right)
$$

In this case, the TIFC operator reduces to the trapezoidal intuitionistic fuzzy weighted averaging operator (TIFWA), i.e.,

$$
\begin{aligned}
& \operatorname{TIFWA}\left(\tilde{a}_{1}, \tilde{a}_{2}, \ldots, \tilde{a}_{n}\right)=\psi\left(\tilde{a}_{1}\right) \tilde{a}_{1} \oplus \psi\left(\tilde{a}_{2}\right) \tilde{a}_{2} \oplus \ldots \\
& \qquad \psi\left(\tilde{a}_{n}\right) \tilde{a}_{n}
\end{aligned}
$$

Proposition 4. Let $\tilde{a}_{i}(i=1,2, \ldots, n)$ be the element in the TIFC operator. If

$$
\psi(B)=\sum_{i=1}^{|B|} \omega_{i} \text { for all } B \subseteq\left\{\tilde{a}_{1}, \ldots, \tilde{a}_{n}\right\}
$$

where $|B|$ is the cardinal number of $B$, then

$$
\omega_{i}=\left(\psi\left(A_{(i)}\right)-\psi\left(A_{(i+1)}\right)\right.
$$

where $\omega_{i} \geqslant 0, i=1, \ldots, n$ and $\sum_{i=1}^{n} \omega_{i}=1$. In this case, the TIFC operator reduces to the trapezoidal intuitionistic fuzzy ordered weighted averaging operator (TIFOWA), i.e., 


$$
\begin{aligned}
& \operatorname{TIFOWA}\left(\tilde{a}_{1}, \tilde{a}_{2}, \ldots, \tilde{a}_{n}\right) \\
& \quad=\omega_{1} \tilde{a}_{(1)} \oplus \tilde{a}_{(2)} \oplus \ldots \oplus \omega_{n} \tilde{a}_{(n)}
\end{aligned}
$$

where $(i)$ indicates a permutation on $X$ such that $\tilde{a}_{(1)} \leqslant \tilde{a}_{(2)} \leqslant \ldots \leqslant \tilde{a}_{(n)}$.

Proposition 5. Let $\tilde{a}_{i}=\left\langle\left(a_{i 1}, a_{i 2}, a_{i 3}, a_{i 4}\right),\left(b_{i 1}, b_{i 2}, b_{i 3}, b_{i 4}\right)\right.$; $\left.\mu_{\tilde{a}_{i}}, v_{\tilde{a}_{i}}\right\rangle \quad(i=1,2, \ldots, n)$ be a collection of trapezoidal intuitionistic fuzzy numbers on $X$ and $\psi$ be a fuzzy measure on $X$. If the values of all $\tilde{a}_{i}$ are identical, i.e., $\tilde{a}_{i}=\tilde{a}=\left\langle\left(a_{1}\right.\right.$, $\left.\left.a_{2}, a_{3}, a_{4}\right),\left(b_{1}, b_{2}, b_{3}, b_{4}\right) ; \mu_{\tilde{a}}, v_{\tilde{a}}\right\rangle$, then the value aggregated by using TIFC operator is as follows

$$
\operatorname{TIFC}\left(\tilde{a}_{1}, \ldots, \tilde{a}_{n}\right)=\tilde{a}
$$

Proof. According to Theorem 2, if $\tilde{a}_{i}=\tilde{a}(i=1,2, \ldots, n)$, we then have

$$
\operatorname{TIFC}\left(\tilde{a}_{1}, \ldots, \tilde{a}_{n}\right)=\left\{\begin{array}{l}
\left(\sum_{i=1}^{n} \tau_{i} a_{1}, \sum_{i=1}^{n} \tau_{i} a_{2}, \sum_{i=1}^{n} \tau_{i} a_{3}, \sum_{i=1}^{n} \tau_{i} a_{4}\right),\left(\sum_{i=1}^{n} \tau_{i} b_{1}, \sum_{i=1}^{n} \tau_{i} b_{2},\right. \\
\left(\sum_{i=1}^{n} \tau_{i} b_{3}, \sum_{i=1}^{n} \tau_{i} b_{4}\right) ; 1-\left(1-\mu_{\tilde{a}}\right)_{i=1}^{n} \tau_{i} \sum_{\left(v_{\tilde{a}}\right)}^{n} \tau_{i}
\end{array}\right\rangle
$$

Note that

$$
\begin{aligned}
& \sum_{i=1}^{n} \tau_{i}=\left[\psi\left(A_{(1)}\right)-\psi\left(A_{(2)}\right)\right]+\left[\psi\left(A_{(2)}\right)-\psi\left(A_{(3)}\right)\right]+\ldots+\left[\psi\left(A_{(n)}\right)-\psi\left(A_{(n+1)}\right)\right] \\
& \quad=\psi\left(A_{(1)}\right)-\psi\left(A_{(n+1)}\right)=1
\end{aligned}
$$

Therefore, one obtains $\operatorname{TIFC}\left(\tilde{a}_{1}, \ldots, \tilde{a}_{n}\right)=\tilde{a}$.

Proposition 6. Let $\tilde{a}_{i}=\left\langle\left(a_{i 1}, a_{i 2}, a_{i 3}, a_{i 4}\right),\left(b_{i 1}, b_{i 2}, b_{i 3}, b_{i 4}\right) ; \mu_{\tilde{a}_{i}}\right.$,

$\left.v_{\tilde{a}_{i}}\right\rangle(i=1,2, \ldots, n)$ be a collection of trapezoidal intuitionistic fuzzy numbers on $X$ and $\psi$ be a fuzzy measure on $X$. Let

$$
\begin{aligned}
& \tilde{a}^{-}=\left\langle\left(\min _{i}\left(a_{i 1}\right), \min _{i}\left(a_{i 2}\right), \min _{i}\left(a_{i 3}\right), \min _{i}\left(a_{i 4}\right)\right),\left(\min _{i}\left(b_{i 1}\right), \min _{i}\left(b_{i 2}\right), \min _{i}\left(b_{i 3}\right), \min _{i}\left(b_{i 4}\right)\right) ; \min _{i}\left(\mu_{\tilde{a}_{i}}\right), \max _{i}\left(v_{\tilde{a}_{i}}\right)\right\rangle \\
& \tilde{a}^{+}=\left\langle\left(\max _{i}\left(a_{i 1}\right), \max _{i}\left(a_{i 2}\right), \max _{i}\left(a_{i 3}\right), \max _{i}\left(a_{i 4}\right)\right),\left(\max _{i}\left(b_{i 1}\right), \max _{i}\left(b_{i 2}\right), \max _{i}\left(b_{i 3}\right), \max _{i}\left(b_{i 4}\right)\right) ; \max _{i}\left(\mu_{\tilde{a}_{i}}\right), \min _{i}\left(v_{\tilde{a}_{i}}\right)\right\rangle
\end{aligned}
$$

Then we have

Proof. It is obvious that $\tilde{a}^{-}$and $\tilde{a}^{+}$are trapezoidal intuitionistic fuzzy numbers. By Theorem 1, we first computed the expected value and score function of $\tilde{a}^{-}, \tilde{a}^{+}$,

$$
\tilde{a}^{-} \leqslant \operatorname{TIFC}\left(\left(\tilde{a}_{1}, \ldots, \tilde{a}_{n}\right) \leqslant \tilde{a}^{+}\right.
$$
and $\operatorname{TIFC}\left(\left(\tilde{a}_{1}, \ldots, \tilde{a}_{n}\right)\right.$ as follows:

$$
\begin{array}{r}
\operatorname{EV}\left(\tilde{a}^{-}\right)=\frac{1}{8}\left[\min _{i}\left(\mu_{\tilde{a}_{i}}\right) \sum_{j=1}^{4} \min _{i}\left(a_{i j}\right)+\left(1-\max _{i}\left(v_{\tilde{a}_{i}}\right)\right) \sum_{j=1}^{4} \min _{i}\left(b_{i j}\right)\right] \\
\operatorname{EV}\left(\tilde{a}^{+}\right)=\frac{1}{8}\left[\max _{i}\left(\mu_{\tilde{a}_{i}}\right) \sum_{j=1}^{4} \max _{i}\left(a_{i j}\right)+\left(1-\min _{i}\left(v_{\tilde{a}_{i}}\right)\right) \sum_{j=1}^{4} \max _{i}\left(b_{i j}\right)\right] \\
\operatorname{EV}\left(\operatorname{TIFC}\left(\tilde{a}_{1}, \ldots, \tilde{a}_{n}\right)\right)=\frac{1}{8}\left[\left(1-\prod_{i=1}^{n}\left(1-\mu_{\tilde{a}_{(i)}}\right)^{\tau_{i}}\right) \sum_{j=1}^{4} \sum_{i=1}^{n} \tau_{i} a_{(i) j}+\left(1-\prod_{i=1}^{n}\left(v_{\tilde{a}_{(i)}}\right)^{\tau_{i}}\right) \sum_{j=1}^{4} \sum_{i=1}^{n} \tau_{i} b_{(i) j}\right] \\
S\left(\tilde{a}^{-}\right)=\operatorname{EV}\left(\tilde{a}^{-}\right) \times\left(\min _{i}\left(\mu_{\tilde{a}_{i}}\right)-\max _{i}\left(v_{\tilde{a}_{i}}\right)\right) \\
S\left(\tilde{a}^{+}\right)=\operatorname{EV}\left(\tilde{a}^{+}\right) \times\left(\max _{i}\left(\mu_{\tilde{a}_{i}}\right)-\min _{i}\left(v_{\tilde{a}_{i}}\right)\right)
\end{array}
$$




$$
\begin{aligned}
& S\left(\operatorname{TIFC}\left(\tilde{a}_{1}, \ldots, \tilde{a}_{n}\right)\right) \\
& =\operatorname{EV}\left(\operatorname{TIFC}\left(\tilde{a}_{1}, \ldots, \tilde{a}_{n}\right)\right) \\
& \quad \times\left(1-\prod_{i=1}^{n}\left(1-\mu_{\tilde{a}_{(i)}}\right)^{\tau_{i}}-\prod_{i=1}^{n}\left(v_{\tilde{a}_{(i)}}\right)^{\tau_{i}}\right)
\end{aligned}
$$

Since $A_{(i)} \supseteq A_{(i+1)}, \tau_{i}=\psi\left(A_{(i)}\right)-\psi\left(A_{(i+1)}\right) \geqslant 0$ holds. Thus, we have

$$
\begin{gathered}
1-\prod_{i=1}^{n}\left(1-\min _{i}\left(\mu_{\tilde{a}_{i}}\right)\right)^{\tau_{i}} \leqslant 1-\prod_{i=1}^{n}\left(1-\mu_{\tilde{a}_{(i)}}\right)^{\tau_{i}} \\
\leqslant 1-\prod_{i=1}^{n}\left(1-\max _{i}\left(\mu_{\tilde{a}_{i}}\right)\right)^{\tau_{i}}
\end{gathered}
$$

and

$$
1-\prod_{i=1}^{n}\left(\max _{i}\left(v_{\tilde{a}_{i}}\right)\right)^{\tau_{i}} \leqslant 1-\prod_{i=1}^{n}\left(v_{\tilde{a}_{(i)}}\right)^{\tau_{i}} \leqslant 1-\prod_{i=1}^{n}\left(1-\min _{i}\left(v_{\tilde{a}_{i}}\right)\right)^{\tau_{i}}
$$

i.e.,

$$
\begin{gathered}
1-\left(1-\min _{i}\left(\mu_{\tilde{a}_{i}}\right) \sum_{i=1}^{n} \tau_{i} \leqslant 1-\prod_{i=1}^{n}\left(1-\mu_{\tilde{a}_{(i)}}\right)^{\tau_{i}}\right. \\
\leqslant 1-\prod_{i=1}^{n}\left(1-\max _{i}\left(\mu_{\tilde{a}_{i}}\right)\right)^{\sum_{i=1}^{n} \tau_{i}}
\end{gathered}
$$

and

$$
\left.\left.1-\max _{i}\left(v_{\tilde{a}_{i}}\right)\right)^{\sum_{i=1}^{n} \tau_{i}} \leqslant 1-\prod_{i=1}^{n}\left(v_{\tilde{a}_{(i)}}\right)^{\tau_{i}} \leqslant 1-\min \left(v_{\tilde{a}_{i}}\right)\right)^{\sum_{i=1}^{n} \tau_{i}}
$$

Note that

$$
\begin{aligned}
\sum_{i=1}^{n} \tau_{i}= & \left(\psi\left(A_{(1)}\right)-\psi\left(A_{(2)}\right)\right)+\left(\psi\left(A_{(2)}\right)-\psi\left(A_{(3)}\right)\right)+\ldots \\
& +\left(\psi\left(A_{(n)}\right)-\psi\left(A_{(n+1)}\right)\right) \\
= & \psi\left(A_{(1)}\right)-\psi\left(A_{(n+1)}\right)=1
\end{aligned}
$$

Thereby, we have

$$
\min _{i}\left(\mu_{\tilde{a}_{i}}\right) \leqslant 1-\prod_{i=1}^{n}\left(1-\mu_{\tilde{a}_{(i)}}\right)^{\tau_{i}} \leqslant \max _{i}\left(\mu_{\tilde{a}_{i}}\right)
$$

and

$$
1-\max _{i}\left(v_{\tilde{a}_{i}}\right) \leqslant 1-\prod_{i=1}^{n}\left(1-v_{\tilde{a}_{(i)}}\right)^{\tau_{i}} \leqslant 1-\min _{i}\left(v_{\tilde{a}_{i}}\right)
$$

On the other hand, note that

$$
\sum_{j=1}^{4} \sum_{i=1}^{n} \tau_{i} \min _{i}\left(a_{i j}\right) \leqslant \sum_{j=1}^{4} \sum_{i=1}^{n} \tau_{i} a_{(i) j} \leqslant \sum_{j=1}^{4} \sum_{i=1}^{n} \tau_{i} \max _{i}\left(a_{i j}\right)
$$

and

$$
\sum_{j=1}^{4} \sum_{i=1}^{n} \tau_{i} \min _{i}\left(b_{i j}\right) \leqslant \sum_{j=1}^{4} \sum_{i=1}^{n} \tau_{i} b_{(i) j} \leqslant \sum_{j=1}^{4} \sum_{i=1}^{n} \tau_{i} \max _{i}\left(b_{i j}\right)
$$

i.e.,

$$
\sum_{j=1}^{4} \min _{i}\left(a_{i j}\right) \leqslant \sum_{j=1}^{4} \sum_{i=1}^{n} \tau_{i} a_{(i) j} \leqslant \sum_{j=1}^{4} \max _{i}\left(a_{i j}\right)
$$

and

$$
\sum_{j=1}^{4} \min _{i}\left(b_{i j}\right) \leqslant \sum_{j=1}^{4} \sum_{i=1}^{n} \tau_{i} b_{(i) j} \leqslant \sum_{j=1}^{4} \max _{i}\left(b_{i j}\right)
$$

Consequently, we have

$$
\operatorname{EV}\left(\tilde{a}^{-}\right) \leqslant \operatorname{EV}\left(\operatorname{TIFC}\left(\tilde{a}_{1}, \ldots, \tilde{a}_{n}\right)\right) \leqslant \operatorname{EV}\left(\tilde{a}^{+}\right)
$$

In view of the above, we have

$$
\min _{i}\left(\mu_{\tilde{a}_{i}}\right) \leqslant 1-\prod_{i=1}^{n}\left(1-\mu_{\tilde{a}_{(i)}}\right)^{\tau_{i}} \leqslant \max _{i}\left(\mu_{\tilde{a}_{i}}\right)
$$

and

$$
1-\max _{i}\left(v_{\tilde{a}_{i}}\right) \leqslant 1-\prod_{i=1}^{n}\left(1-v_{\tilde{a}_{(i)}}\right)^{\tau_{i}} \leqslant 1-\min _{i}\left(v_{\tilde{a}_{i}}\right)
$$

Thus

$$
\min _{i}\left(\mu_{\tilde{a}_{i}}\right)-\max _{i}\left(v_{\tilde{a}_{i}}\right) \leqslant 1-\prod_{i=1}^{n}\left(1-\mu_{\tilde{a}_{(i)}}\right)^{\tau_{i}}-\prod_{i=1}^{n}\left(v_{\tilde{a}_{(i)}}\right)^{\tau_{i}}
$$

$$
\leqslant \max _{i}\left(\mu_{\tilde{a}_{i}}\right)-\min _{i}\left(v_{\tilde{a}_{i}}\right)
$$

As a result, we have

$\operatorname{EV}\left(\tilde{a}^{-}\right)$

$$
\begin{aligned}
& \times\left(\min _{i}\left(\mu_{\tilde{a}_{i}}\right)-\max _{i}\left(v_{\tilde{a}_{i}}\right)\right) \leqslant \operatorname{EV}\left(\operatorname{TIFC}\left(\tilde{a}_{1}, \ldots, \tilde{a}_{n}\right)\right) \\
& \times\left(1-\prod_{i=1}^{n}\left(1-\mu_{\tilde{a}_{(i)}}\right)^{\tau_{i}}-\prod_{i=1}^{n}\left(v_{\tilde{a}_{(i)}}\right)^{\tau_{i}}\right) \leqslant \operatorname{EV}\left(\tilde{a}^{+}\right)
\end{aligned}
$$

$$
\times\left(\max _{i}\left(\mu_{\tilde{a}_{i}}\right)-\min _{i}\left(v_{\tilde{a}_{i}}\right)\right)
$$

this can be further written as

$$
S\left(\tilde{a}^{-}\right) \leqslant S\left(\operatorname{TIFC}\left(\tilde{a}_{1}, \ldots, \tilde{a}_{n}\right) \leqslant S\left(\tilde{a}^{+}\right)\right.
$$

According to Definition 5, we have

$$
\tilde{a}^{-} \leqslant \operatorname{TIFC}\left(\tilde{a}_{1}, \ldots, \tilde{a}_{n}\right) \leqslant \tilde{a}^{+}
$$

This implies that Eq. (29) holds. This completes the proof of the proposition. 


\section{Multi-criteria decision making method based on trapezoidal intuitionistic fuzzy Choquet integral operator}

Multi-criteria decision making is the process of choosing the best alternative from some feasible alternatives based on multiple criteria. In each of these decisions, decision makers have several criteria to consider. Generally, in real decision-making problems, much information available to decision makers is vague and imprecise. Trapezoidal intuitionistic fuzzy numbers can effectively deal with uncertain and vague information in real applications. In the following, we fist describe the multi-criteria decision making problems with trapezoidal intuitionistic fuzzy setting.

For a multi-criteria decision making problem, let $A=\left\{a_{1}\right.$, $\left.a_{2}, \ldots, a_{m}\right\}$ be a set of alternatives, $C=\left\{c_{1}, c_{2}, \ldots, c_{n}\right\}$ are $n$ decision criteria. Assume the preference value of alternative $a_{i}$ on the criteria $c_{j}$ is a trapezoidal intuitionistic fuzzy number $t_{i j}=\left\langle\left(a_{i j 1}, a_{i j 2}, a_{i j 3}, a_{i j 4}\right),\left(b_{i j 1}, b_{i j 2}, b_{i j 3}, b_{i j 4}\right) ; \mu_{t_{i j}}\right.$, $\left.v_{t_{i j}}\right\rangle(i=1,2, \ldots, m$ and $j=1,2, \ldots, n)$, thus the characteristic of alternatives can be expressed by the intuitionistic fuzzy numbers.

To handle this multi-criteria decision making problems with trapezoidal intuitionistic fuzzy setting, we propose the following procedure:

Step 1 . With the preference value of alternative $a_{i}(i=$ $1,2, \ldots, m)$ on the criteria $c_{j}(j=1,2, \ldots, n)$ which is described by a trapezoidal intuitionistic fuzzy number $\tilde{t}_{i j}=\left\langle\left(a_{i j 1}, a_{i j 2}\right.\right.$, $\left.\left.a_{i j 3}, a_{i j 4}\right),\left(b_{i j 1}, b_{i j 2}, b_{i j 3}, b_{i j 4}\right) ; \mu_{t_{i j}}, v_{t_{i j}}\right\rangle(i=1,2, \ldots, m$ and $j=$ $1,2, \ldots, n)$, the decision matrix $D=\left(t_{i j}\right)_{m \times n}$ can be obtained.

Step 2. Standardize decision matrix $D$. One can obtain the normalized decision matrix $D^{\prime}=\left(\tilde{r}_{i j}\right)_{m \times n}$ using the following transformation.

$$
\tilde{r}_{i j}=\left\langle\left(\frac{a_{i j 1}}{\theta_{j}^{+}}, \frac{a_{i j 2}}{\theta_{j}^{+}}, \frac{a_{i j 3}}{\theta_{j}^{+}}, \frac{a_{i j 4}}{\theta_{j}^{+}}\right),\left(\frac{b_{i j 1}}{\theta_{j}^{+}}, \frac{b_{i j 2}}{\theta_{j}^{+}}, \frac{b_{i j 3}}{\theta_{j}^{+}}, \frac{b_{i j 4}}{\theta_{j}^{+}}\right) ; \mu_{\tilde{t}_{i j}}, v_{t_{i j}}\right\rangle
$$$$
j \in \Omega_{B}
$$

$$
\tilde{r}_{i j}=\left\langle\left(\frac{\theta_{j}^{-}}{a_{i j 4}}, \frac{\theta_{j}^{-}}{a_{i j 3}}, \frac{\theta_{j}^{-}}{a_{i j 2}}, \frac{\theta_{j}^{-}}{a_{i j 1}}\right),\left(\frac{\theta_{j}^{-}}{b_{i j 4}}, \frac{\theta_{j}^{-}}{b_{i j 3}}, \frac{\theta_{j}^{-}}{b_{i j 2}}, \frac{\theta_{j}^{-}}{b_{i j 1}}\right) ; \mu_{\tilde{t}_{i j}}, v_{t_{i j}}\right\rangle
$$

$$
j \in \Omega_{C}
$$

where $\theta_{j}^{+}=\max _{i}\left(b_{i j 4}\right), \theta_{j}^{-}=\min _{i}\left(b_{i j 1}\right)$, and $\Omega_{B}$ and $\Omega_{C}$ are the benefit and cost criteria, respectively.

Step 3. For each alternative $a_{i}(i=1, \ldots, m)$, according to Definition 5 , by score functions $S$ and accuracy functions $H$, rank the normalized preference value $\tilde{r}_{i j}$ of alternative $a_{i}(i=1, \ldots, m)$ on the criteria $c_{j}(j=1,2, \ldots, n)$ such that $\tilde{r}_{i(j)} \leqslant$ $\tilde{r}_{i(j+1)}$.

Step 4. Confirm the fuzzy measures on criteria of $C$. There are many methods that can be used to obtain fuzzy measures, such as linear methods (Marichal \& Roubens, 1998), quadratic methods (Grabisch, 1996a, 1996b), statistics and neural networks methods (Wang, 1995; Wang, Klir, \& Wang, 1998).

Step 5. Aggregate the normalized preference value $\tilde{r}_{i j}$ in the $i$ th line of normalized decision matrix $D^{\prime}=\left(\tilde{r}_{i j}\right)_{m \times n}$ into an overall normalized preference value $\tilde{a}_{i}=\left\langle\left(a_{i 1}, a_{i 2}, a_{i 3}\right.\right.$, $\left.\left.a_{i 4}\right),\left(b_{i 1}, b_{i 2}, b_{i 3}, b_{i 4}\right) ; \mu_{\tilde{a}_{i}}, v_{\tilde{a}_{i}}\right\rangle(i=1,2, \ldots, m)$ by using trapezoidal intuitionistic fuzzy Choquet integral operator:

$$
\operatorname{TIFC}\left(\tilde{r}_{i 1}, \ldots, \tilde{r}_{i n}\right)=\bigoplus_{i=1}^{n} \tilde{r}_{i(j)}\left[\psi\left(A_{(j)}\right)-\psi\left(A_{(j+1)}\right)\right]
$$

where $(j)$ indicates a permutation such that $\tilde{a}_{(1)} \leqslant \tilde{a}_{(2)} \leqslant \ldots \leqslant$ $\tilde{a}_{(n)}$. In addition, $A_{(j)}=\left\{c_{(j)}, \ldots, c_{(n)}\right\}, A_{(n+1)}=\phi$.

Step 6. According to Definition 5, compute the score function $S\left(\tilde{a}_{i}\right)$ and accuracy function $H\left(\tilde{a}_{i}\right)$, then compare the overall normalized preference value $\tilde{a}_{i}(i=1,2, \ldots, m)$ related to alternative $a_{i}(i=1,2, \ldots, m)$. The alternative with the biggest $\tilde{a}_{i}$ can be considered as the best alternative.

\section{Illustrative examples}

In this section, we apply the procedure that is proposed in the previous sections to the multi-criteria decision-making problem of computer software selection. It is assumed that a computer center wants to select a new information system to enhance work efficiency. Now suppose that after carefully consideration, there are three alternatives $\left\{a_{1}, a_{2}\right.$, $\left.a_{3}\right\}$ of which core competences can be evaluated by the following criteria:

(1) costs of software and hardware $\left(c_{1}\right)$;

(2) contribution to organizational performance improvement $\left(c_{2}\right)$; and

(3) outsourcing software developer's reliability $\left(c_{3}\right)$.

Step 1. A group of experts are invited to consider these alternatives $\left\{a_{1}, a_{2}, a_{3}\right\}$. After careful analysis of their characteristics, the experts give the following decision matrix $D=\left(\tilde{t}_{i j}\right)_{3 \times 3}$ :

$$
\begin{aligned}
& \tilde{t}_{11}=\langle(0.5,0.6,0.7,0.8),(0.4,0.6,0.7,0.8) ; 0.7,0.2\rangle \\
& \tilde{t}_{21}=\langle(0.3,0.4,0.5,0.6),(0.2,0.4,0.5,0.7) ; 0.4,0.3\rangle \\
& \tilde{t}_{31}=\langle(0.4,0.5,0.6,0.7),(0.3,0.4,0.6,0.8) ; 0.5,0.3\rangle \\
& \tilde{t}_{12}=\langle(0.6,0.7,0.8,0.9),(0.5,0.7,0.8,0.9) ; 0.8,0.1\rangle \\
& \tilde{t}_{22}=\langle(0.2,0.3,0.5,0.6),(0.2,0.3,0.5,0.6) ; 0.7,0.1\rangle \\
& \tilde{t}_{32}=\langle(0.2,0.4,0.5,0.6),(0.1,0.3,0.5,0.6) ; 0.7,0.2\rangle
\end{aligned}
$$




$$
\begin{aligned}
& \tilde{t}_{13}=\langle(0.3,0.4,0.5,0.6),(0.3,0.4,0.5,0.7) ; 0.4,0.5\rangle \\
& \tilde{t}_{23}=\langle(0.5,0.6,0.7,0.8),(0.4,0.6,0.7,0.8) ; 0.5,0.2\rangle \\
& \tilde{t}_{33}=\langle(0.6,0.7,0.8,0.9),(0.6,0.7,0.8,0.9) ; 0.7,0.2\rangle \\
& \tilde{r}_{11}=\langle(0.25,0.2857,0.3333,0.4),(0.25,0.2857,0.3333,0.5) ; 0.7,0.2\rangle \\
& \tilde{r}_{21}=\langle(0.3333,0.4,0.5,0.6667),(0.2857,0.4,0.5,0.1) ; 0.4,0.3\rangle \\
& \tilde{r}_{31}=\langle(0.2857,0.3333,0.4,0.5),(0.25,0.3333,0.5,0.6667) ; 0.5,0.3\rangle \\
& \tilde{r}_{12}=\langle(0.6667,0.7778,0.8889,1),(0.5556,0.7778,0.8889,1) ; 0.8,0.1\rangle \\
& \tilde{r}_{22}=\langle(0.2222,0.3333,0.5556,0.6667),(0.2222,0.3333,0.5556,0.6667) ; 0.7,0.1\rangle \\
& \tilde{r}_{32}=\langle(0.2222,0.4444,0.5556,0.6667),(0.1111,0.3333,0.5556,0.6667) ; 0.7,0.2\rangle \\
& \tilde{r}_{13}=\langle(0.3333,0.4444,0.5556,0.6667),(0.3333,0.4444,0.5556,0.7778) ; 0.4,0.5\rangle \\
& \tilde{r}_{23}=\langle(0.5556,0.6667,0.7778,0.8889),(0.4444,0.6667,0.7778,0.8889) ; 0.5,0.2\rangle \\
& \tilde{r}_{33}=\langle(0.6667,0.7778,0.8889,1),(0.6667,0.7778,0.88889,1) ; 0.7,0.2\rangle
\end{aligned}
$$

Step 3. For each alternative $a_{i}(i=1,2,3)$, according to Definition 5 , the normalized preference value $\tilde{r}_{i j}$ is reordered such that $r_{i j} \leqslant r_{i(j+1)}$, as follows:

$$
\begin{gathered}
\tilde{r}_{1(1)}=\tilde{r}_{13}, \tilde{r}_{1(2)}=\tilde{r}_{11}, \tilde{r}_{1(3)}=\tilde{r}_{12}, \\
\tilde{r}_{2(1)}=\tilde{r}_{21}, \tilde{r}_{2(2)}=\tilde{r}_{23}, \tilde{r}_{2(3)}=\tilde{r}_{22}, \\
\tilde{r}_{3(1)}=\tilde{r}_{31}, \tilde{r}_{3(2)}=\tilde{r}_{32}, \tilde{r}_{3(3)}=\tilde{r}_{33} .
\end{gathered}
$$

Step 4. Suppose the fuzzy measures on criteria of $C$ as follows:

$$
\psi\left(c_{1}\right)=0.4, \psi\left(c_{2}\right)=0.3, \psi\left(c_{3}\right)=0.3, \psi\left(c_{1}, c_{2}\right)
$$$$
=0.8, \psi\left(c_{1}, c_{3}\right)=0.6, \psi\left(c_{2}, c_{3}\right)=0.7, \psi\left(c_{1}, c_{2}, c_{3}\right)=1 \text {. }
$$

Step 5. According to Eqs. (21) and (22), aggregate the normalized preference value $\tilde{r}_{i j}$ in the $i$ th line of normalized decision matrix $D^{\prime}=\left(\tilde{r}_{i j}\right)_{m \times n}$ into an overall normalized preference value $\tilde{a}_{i}=\left\langle\left(a_{i 1}, a_{i 2}, a_{i 3}, a_{i 4}\right),\left(b_{i 1}, b_{i 2}, b_{i 3}, b_{i 4}\right) ; \mu_{\tilde{a}_{i}}\right.$, $\left.v_{\tilde{a}_{i}}\right\rangle(i=1,2,3)$ by using trapezoidal intuitionistic fuzzy Choquet integral operator:

$$
\begin{gathered}
\tilde{a}_{1}=\langle(0.7333,0.8847,1.0889,1.2867),(0.7,0.8847,1.0889,1.4778) ; 0.8587,0.0692\rangle \\
\tilde{a}_{2}=\langle(0.7889,0.9667,1.2111,1.4892),(0.6364,0.9667,1.2111,1.8222) ; 0.7426,0.0487\rangle
\end{gathered}
$$




$$
\tilde{a}_{3}=\langle(0.6143,0.8778,1.0556,1.2667),(0.5278,0.8,1.1556,1.4334) ; 0.85,0.06\rangle
$$

Step 6. According to Definition 5, compute the score function $S\left(\tilde{a}_{i}\right)(i=1,2,3)$ :

$$
S\left(\tilde{a}_{1}\right)=0.7198, S\left(\tilde{a}_{2}\right)=0.6696, S\left(\tilde{a}_{3}\right)=0.6837 \text {. }
$$

Then, we can obtain

$$
\tilde{a}_{1}>\tilde{a}_{3}>\tilde{a}_{2}
$$

Therefore, the order of the alternatives is $a_{1}, a_{3}, a_{2}$. Hence the best alternative is $a_{1}$.

\section{Conclusions}

As an extension of intuitionistic triangular fuzzy numbers and intuitionistic fuzzy sets, intuitionistic trapezoidal fuzzy numbers can express more adequate and flexible information and thus have a stronger expression ability to deal with the uncertain information. Most existing studies usually consider the situations with independent criteria. However, for real decision-making problems, there always exist interactive characteristics among the elements in an uncertain situation. In this paper, a trapezoidal intuitionistic fuzzy aggregation operator based on the Choquet integral is proposed for multi-criteria decision-making problems, in which the preference values for an alternative are expressed by trapezoidal intuitionistic fuzzy numbers. The proposed operator has the ability to deal with interaction information among decision making criteria. It is shown that the trapezoidal intuitionistic fuzzy weighted averaging operator (TIFWA) and the trapezoidal intuitionistic fuzzy ordered weighted averaging operator (TIFOWA) are special cases of the operator proposed in this paper. In addition, two operational laws and the expected value of the trapezoidal intuitionistic fuzzy number are introduced and some of properties of the proposed operator are investigated. An algorithm to tackle the multi-criteria decision making problems with trapezoidal intuitionistic fuzzy setting has been presented. For illustration purposes, the proposed procedure has been applied to a computer software selection problem.

Although the example has shown the stronger capability of the trapezoidal intuitionistic fuzzy numbers and some of their operators to deal with the uncertain information, they should be further illustrated in some actual fields, such as pattern recognition and medical diagnosis. We expect some new operators on trapezoidal intuitionistic fuzzy numbers to be investigated in future studies.

Acknowledgements This research was supported by the National Natural Science Foundation of China (Nos. 71431006, 71401184), Key Project of Philosophy and Social Sciences Research, Ministry of Education, PRC (No. 13JZD0016), China Postdoctoral Science Foundation (No. 2014M552169) and Central South University Business Management Postdoctoral Research Station.

\section{References}

Abbasbandy, S., \& Hajjari, T. (2009). A new approach for ranking of trapezoidal fuzzy number. Computers \& Mathematics with Applications (Oxford, England), 57, 413-419

Asady, B., \& Zendehnam, A. (2007). Ranking fuzzy numbers by distance minimization. Applied Mathematical Modelling, 31, 2589-2598

Atanassov, K. (1986). Intuitionistic fuzzy sets. Fuzzy Sets and Systems, 20, 87-96

Chen, S., \& Tan, J. (1994). Handling multicriteria fuzzy decision-making problems based on vague set theory. Fuzzy Sets and Systems, 67, 163172

Choquet, G. (1954). Theory of Capacities. Annales de l'Institut Fourier, $5,131-295$

Dubois, D., \& Prade, H. (1980). Fuzzy Sets and Systems: Theory and Application. New York: Academic Press

Grabisch, M. (1996a). The representation of importance and interaction of features by fuzzy measures. Pattern Recognition Letters, 17, 567575

Grabisch, M. (1996b). The application of fuzzy integrals in multicriteria decision making. European Journal of Operational Research, 89, 445-456

Höhle, U. (1982). Integration with respect to fuzzy measures. In Anon. (Eds.), Proceedings of the IFAC Symposium on Theory and Application of Digital Control (pp. 35-37). New Delhi, India

Hong, D., \& Choi, C. (2000). Multicriteria fuzzy decision-making problems based on vague set theory. Fuzzy Sets and Systems, 114, 103-113

Li, D. (2005). Multiattribute decision making models and methods using intuitionistic fuzzy sets. Journal of Computer and System Sciences, 70, 73-85

Li, D., Wang, Y., Liu, S., \& Shan, F. (2008). Fractional programming methodology for multi-attribute group decision making using IFS. Applied Soft Computing, 8, 219-225

Liu, H., \& Wang, G. (2007). Multi-criteria decision-making methods based on intuitionistic fuzzy sets. European Journal of Operational Research, 179, 220-233

Marichal, J., \& Roubens, M. (1998). Dependence between criteria and multiple criteria decision aid. In 2nd International Workshop on Preferences and Decisions, Trento, Italy

Murofushi, T., \& Sugeno, M. (1989). An interpretation of fuzzy measure and the Choquet integral as an integral with respect to a fuzzy measure. Fuzzy Sets and Systems, 29, 201-227

Murofushi, T., \& Sugeno, M. (1991). A theory of fuzzy measuresrepresentations, the Choquet integral, and null sets. Journal of Mathematical Analysis and Applications, 159, 532-549

Nehi, H., \& Maleki, H. (2005). Intuitionistic fuzzy numbers and it's applications in fuzzy optimization problem. In Proceedings of the 9th WSEAS International Conference on Systems, Athens, Greece, 1-5

Sugeno, M. (1974). Theory of fuzzy integral and its application (Doctorial dissertation). Tokyo: Tokyo Institute of Technology

Tan, C., \& Chen, X. (2010). Intuitionistic fuzzy Choquet integral 
operator for multi-criteria decision making. Expert Systems with Applications, 37, 149-157

Hadi-Vencheh, A., \& Allame, M. (2010). On the relation between a fuzzy number and its centroid. Computers \& Mathematics with Applications (Oxford, England), 59, 3578-3582

Wang, J. (1995). Determining fuzzy measures by using statistics and neural networks, In Anon. (Eds.) Proc. of IFSA'95, Sao Paulo

Wang, J., \& Zhang, Z. (2009). Aggregation operators on intuitionistic trapezoidal fuzzy number and its application to multicriteria decision making problems. Journal of Systems Engineering and Electronics, 20, 321-326

Wang, Z., Klir, G., \& Wang, J. (1998). Neural networks used for determining belief measures and plausibility measures. Intelligent Automation and Soft Computing, 4, 313-324

Wei, G. (2009). Some geometric aggregation functions and their application to dynamic multiple attribute decision making in the intuitionistic fuzzy setting. International Journal of Uncertainty, Fuzziness and Knowledge-based Systems, 17, 179-196

Wu, J., \& Zhang, Q. (2011). Multicriteria decision making method based on intuitionistic fuzzy weighted entropy. Expert Systems with Applications, 38, 916-922

$\mathrm{Xu}$, Z. (2007). Methods for aggregating interval-valued intuitionistic fuzzy information and their application to decision making. Control and Decision, 22, 215-219 [in Chinese]

$\mathrm{Xu}$, Z. (2010). Choquet integrals of weighted intuitionistic fuzzy information. Information Sciences, 180, 726-736

Xu, Z., \& Yager, R. (2006). Some geometric aggregation operators based on intuitionistic fuzzy sets. International Journal of General Systems, 35, 417-433

Xu, Z., \& Yager, R. (2008). Dynamic intuitionistic fuzzy multi-attribute decision making. International Journal of Approximate Reasoning, 48, 246-262

Yager, R.R. (1988). On ordered weighted averaging aggregation operators in multi-criteria decision making. IEEE Transactions on Systems, Man, and Cybernetics, 18, 183-190

Ye, J. (2009). Multicriteria fuzzy decision-making method based on a novel accuracy function under interval-valued intuitionistic fuzzy environment. Expert Systems with Applications, 36, 6899-6902

Ye, J. (2010). Fuzzy decision-making method based on the weighted correlation coefficient under intuitionistic fuzzy environment. European Journal of Operational Research, 205, 202-204

Ye, J. (2011). Expected value method for intuitionistic trapezoidal fuzzy multicriteria decision-making problems. Expert Systems with Applications, 03, 059 